\title{
A Two-Stage Heat Transfer Model for the Peripheral Layers of a Grain Store
}

\author{
ALEXSANDAR ANTIC $\dagger$ \\ aa07@uow.edu.au \\ School of Mathematics \& Applied Statistics, University of Wollongong, Wollongong, \\ NSW, Australia \\ JAMES M. HILL \\ jhill@uow.edu.au \\ School of Mathematics \& Applied Statistics, University of Wollongong, Wollongong, \\ NSW, Australia
}

\begin{abstract}
An understanding of the flow of heat in grain store structures, in particular, within the peripheral layer, is important from many industrial perspectives. To analyse the heat transfer within such regions a mathematical model known as the two-stage heat transfer model is proposed. This model makes a distinction between the air and grain within the grain bulk, and thus takes into consideration the fact that the rate of heat transfer through the grain is different to that through the interstitial air surrounding the grain. Such a model lends itself to a solution via Laplace transforms and approximate analytical results are obtained for small and large times. In addition, the Stehfest numerical algorithm is used for the inversions and very good agreement is obtained between the two approaches. The present model is compared to a previously developed double-diffusivity heat transfer model by the authors, and good agreement is obtained. At present, no experimental data is available to validate the model as it is very difficult to measure the air and grain temperatures separately, particularly in the peripheral layer. The proposed model provides insight into the potential difference existing between the air and grain temperatures.
\end{abstract}

Keywords: heat transfer, two-stage, double-diffusivity, peripheral layer, grain.

\section{Introduction}

An understanding of the flow of heat within a grain store is very important from many industrial perspectives. The peripheral layer, the $150 \mathrm{~mm}-$ $200 \mathrm{~mm}$ layer of grain in contact with the store structure or the headspace air, is of particular importance as many grain storage problems occur there, including:

- caking, the formation of crust or mould, on the surface of the grain bulk,

$\dagger$ Requests for reprints should be sent to chool of Mathematics \& Applied Statistics, University of Wollongong, Wollongong, NSW, Australia. 
- aeration, the pumping of ambient air through a static grain bulk, does not influence temperature and relative humidity within these regions,

- passive heat disinfestation may occur within such regions when exposed to long periods of direct sunlight,

- appearance of insect pests, such as Psocids, which are causing considerable problems for the Australian grain industry.

In this paper we model the heat transfer that occurs in such regions, and in particular within the peripheral layer alongside the vertical grain store wall, where there occur considerable diurnal temperature fluctuations. Many problems occur here, so an understanding of the difference between the air and grain temperatures is very important. For instance, with Psocids, their microclimate determines where they dwell, so that an understanding of the temperature on the surface of the grain kernel, and its vicinity, are particularly important.

Our analysis is based on a mathematical model of temperature profiles within such regions. Accordingly, a mathematical model known as the twostage heat transfer model, and based on McNabb [10], is proposed. This model predicts the heat transfer in the peripheral layer and differentiates between the air and grain, thus taking into consideration the fact that the heat transfer in the grain is different to that in the surrounding air, allowing us to predict the air and grain temperatures separately.

Currently, there is no experimental data available to validate the model as it is difficult to measure the air and grain temperatures separately, particularly in the peripheral layer. The proposed model will at least provide some insight into the differences between the two temperatures. This will assist in solving some of the problems faced by the grain industry, such as predicting the most likely habitat of the Psocids, thus leading to potentially more efficient insect control.

In the next section a brief literature review is presented of some of the the main results in the area of double-porosity pertaining to this work. In section 3 the two-stage heat transfer model is formulated. In Section 4 Laplace transforms are used to find a semi-analytical solution to the model. In Section 4.1 approximate analytical solutions are obtained for the inverse Laplace transforms for small and large time. In Section 4.2 some results are presented by using the Stehfest [15] algorithm to obtain the inversions numerically. In Section 5 the present two-stage model is compared to a previously developed double-diffusivity heat transfer model by Antic and Hill [1]. 


\section{Literature Review}

Similar double-porosity models exist for the modelling of the propagation of pressures in a porous medium, in particular within the groundwater, geothermal and petroleum industries. McGuinness [9] extended McNabb's double-porosity model for the pressure response of a naturally fractured reservoir of single-phase fluid to allow for blocks of varying sizes. Moench [12], in his study of doubly-porous groundwater reservoirs, incorporated the effects of a thin layer of low permeable material or fracture skin which may be present at fracture-block interfaces. Babcock et al. [2] developed a model for longitudinal dispersion mechanisms during steady flow of a fluid through unconsolidated spherical beads which include a stagnant fluid film surrounding the particle. Skopp and Warrick [14] studied miscible displacement by considering there to be a mobile phase and a stationary phase, whereby solute transfer occurs through the mobile phase via convection, and through the stationary phase via diffusion.

Vortmeyer and Schaefer [19] formulated a dual-region model for an adiabatic packed bed with gas flowing through it. The main difference between this model and the two-stage model proposed in this paper is that it uses the same coordinate system to describe both the gas and solid phases, and hence assumes an average temperature for the solid phase rather than considering the specific geometry of the solid phase and thus allowing the temperature to be determined at various points within.

Pruess' [13] MINC method is conceptually similar and is a generalisation of the double-porosity concept originally proposed by Barenblatt [3] and introduced into petroleum literature by Warren and Root [20], among others, in the form of the double-porosity model. The MINC method is a numerical method for simulating transient nonisothermal, two-phase flow of water in a fractured porous medium.

Vargas and McCarthy [17] developed a novel Thermal Particle Dynamics (TDP) simulation technique which incorporates both contact mechanics and contact conductance theories in order to model the dynamics of flow and heat conductance through granular materials.

Davis [6] used a two-stage approach based on McNabb [10] to model pyritic oxidation within a waste rock dump, where it is assumed that oxygen transport is the rate-limiting step in the oxidation process. He considered the difference in predictions between a dump assumed to comprise of particles of a single or average size, and a dump where a range of particle sizes is considered. 


\section{The Two-Stage Heat Transfer Model}

The two-stage heat transfer model is adopted from McNabb [10] in order to solve the problem of heat transfer in a grain store. McNabb [10] first proposed a two-stage model for the modelling of the propagation of pressures in a porous medium with its permeability affected by a homogeneous distribution of fissures which effectively partition the medium into blocks of a particular geometry. This results in there being two pressures associated with the medium, one with the fissures, and one with the blocks.

McNabb observed that in order to model such systems in their most general form the problem of dimensionality must be confronted. Three variables are needed to locate the block within the system, and then another four variables, three of space and one of time, are required to describe the block itself. For certain cases of these problems a factorisation can be achieved such that the two problems, the fissures and the blocks, can be essentially uncoupled. This then lends itself to a method of solution via Laplace transforms.

The two-stage model is based on the assumption that in the neighbourhood of every point $x$ in the fissure system, there is associated a block, and within each block there is associated a specific geometry with a different spatial coordinate system $y$. Hence, the model composes of two diffusion equations, one for the pressure change in the fissures, and another for the pressure change in the blocks. These two diffusion equations are coupled together by the changes in fissure pressure acting as a boundary condition for the blocks located in its neighbourhood.

It is assumed that the fissure permeability is high enough compared to the block permeability so that the pressure drop in the fissure system across a typical block is negligible. Furthermore, it is assumed that the fissure system is in contact with the block system everywhere, that is, it is continuous, with pressure changes reaching the blocks only via the fissure system, and with the blocks acting as distributed sources of fluid to the fissure system. Thus, the corresponding equations are:

$$
\begin{gathered}
\phi_{f} \mu c \frac{\partial P_{f}}{\partial t}=K_{f} \frac{\partial^{2} P_{f}}{\partial x^{2}}+F(x, t), \\
\phi_{b} \mu c \frac{\partial P_{b}}{\partial t}=K_{b} \frac{\partial^{2} P_{b}}{\partial y^{2}}, \quad y \epsilon V_{x}, \\
F(x, t)=\frac{K_{b}}{V_{x}} \iint_{S_{x}} \frac{\partial P_{b}}{\partial y} \cdot d \sigma, \quad y \epsilon S_{x}, \\
P_{b}(x, y, t)=P_{f}(x, t), \quad y \epsilon S_{x},
\end{gathered}
$$


This model is sometimes referred to as the fruitcake model because it is analogous to modelling diffusion in a fruitcake which consists of a cake mix (or highly permeable fissures) and the fruit (or less permeable blocks).

To adopt the McNabb approach for the grain store heat transfer problem, we begin by making the assumption that the grains are all uniform in size, and that they are spherical in shape. This is a legitimate assumption to make, in particular for the case of canola, which is currently of particular interest to the Australian grain industry. We also assume that convective currents are negligible as it is observed that there is effectively no air movement within the peripheral layer. Thus, the one-dimensional two-stage heat transfer model is:

$$
\begin{gathered}
\frac{\partial T_{a}}{\partial t}=K_{a} \frac{\partial^{2} T_{a}}{\partial x^{2}}-\frac{F(x, t)}{\rho_{a} c_{a}}, \\
\frac{\partial T_{g}}{\partial t}=K_{g}\left(\frac{\partial^{2} T_{g}}{\partial r^{2}}+\frac{2}{r} \frac{\partial T_{g}}{\partial r}\right), \\
F(x, t)=\frac{3 \kappa_{g}\left(1-\phi_{a}\right)}{r} \frac{\partial T_{g}}{\partial r}, \quad r=a, \\
T_{g}(x, r, t)=T_{a}(x, t), \quad r=a,
\end{gathered}
$$

with the following initial and boundary conditions:

$$
T_{a}(x, 0)=T_{a 0}, \quad T_{g}(x, r, 0)=T_{g 0}, \text { and } T_{a}(0, t)=T_{b},
$$

where $T_{a}(x, t)$ represents the temperature in the air at position $x$ and at time $t$, and $T_{g}(x, r, t)$ represents the temperature in the grain at position $r$ at time $t$, and in the neighbourhood of $x$.

Equation (5) describes the temperature in the air, with a sink term $F$, equation (6) describes the temperature in the grain, equation (7) is the sink term, describing the transfer of heat from the air to the grain through the surface of the grain, and equation (8) is a continuity boundary condition stating that on the surface of the grain, the air and grain temperatures are in equilibrium. The model distinguishes between the air and grain whereby heat flows through the air to the grains, and is then transferred into the grain through its surface. The actual grain geometry is taken into consideration with a separate spatial coordinate system used to represent the temperature at various points within each grain kernel. Hence, there are two separate spatial coordinate systems used, one for the grain bulk (macroscopic) and one for the grain kernel (microscopic). 


\section{A Solution Via Laplace Transforms}

The two-stage model lends itself well to a solution technique involving Laplace transforms. The method of solution is as follows: We begin by first solving (6) for $T_{g}$ using (8) as the boundary condition. We then calculate (7) from the previously calculated value of $T_{g}$, which gives an expression for the sink term $F$. We then substitute this into (5) and finally solve for $T_{a}$.

We begin by taking Laplace transforms with respect to time and then use a factorisation approach in Laplace transform space which allows us to separate into functions of $(x, s)$ and $(r, s)$. Upon taking Laplace transforms with respect to time of (5)-(8), and assuming zero initial conditions, we have

$$
\begin{gathered}
\frac{\partial^{2} \bar{T}_{a}}{\partial x^{2}}=\frac{1}{K_{a}}\left(s \bar{T}_{a}+\frac{\bar{F}(x, s)}{\rho_{a} c_{a}}\right), \\
\frac{1}{r^{2}} \frac{\partial}{\partial r}\left(r^{2} \frac{\partial \bar{T}_{g}}{\partial r}\right)=\frac{s}{K_{g}} \bar{T}_{g}, \\
\bar{F}(x, s)=\frac{3 \kappa_{g}\left(1-\phi_{a}\right)}{a} \frac{\partial \bar{T}_{g}}{\partial r}(x, a, s), \\
\bar{T}_{g}(x, a, s)=\bar{T}_{a}(x, s),
\end{gathered}
$$

where

$$
\begin{aligned}
\bar{T}_{a}(x, s) & =L\left\{T_{a}(x, t)\right\}=\int_{0}^{\infty} e^{-s t} T_{a}(x, t) d t, \\
\bar{T}_{g}(x, r, s) & =L\left\{T_{g}(x, r, t)\right\}=\int_{0}^{\infty} e^{-s t} T_{g}(x, r, t) d t .
\end{aligned}
$$

We now assume a factorisation. The physical significance of this factorisation is that the structure of the grain is independent of its position within the grain bulk. The mathematical significance is simply that we can separate into functions of $x$ and $r$. The factorisation is as follows:

$$
\bar{T}_{g}(x, r, s)=\bar{T}_{a}(x, s) \phi(r, s),
$$

where $\phi(r, s)$ is such that

$$
\begin{gathered}
\frac{1}{r^{2}} \frac{\partial}{\partial r}\left(r^{2} \frac{\partial \phi}{\partial r}\right)=\frac{s}{K_{g}} \phi, \quad r<a, \\
\phi=1, \quad r=a,
\end{gathered}
$$




$$
\lim _{r \rightarrow 0}\left(r^{2} \frac{\partial \phi}{\partial r}\right)=0 .
$$

The solution to (14) can easily be found to be

$$
\phi(r, s)=\frac{a}{r} \frac{\sinh \left(r \sqrt{s / K_{g}}\right)}{\sinh \left(a \sqrt{s / K_{g}}\right)} .
$$

Hence, (11) becomes

$$
\bar{F}(x, s)=\frac{3 \kappa_{g}\left(1-\phi_{a}\right)}{a} \bar{T}_{a}(x, s) \frac{\partial \phi}{\partial r}(a, s) .
$$

Now,

$$
\frac{\partial \phi}{\partial r}=\frac{a \sqrt{s / K_{g}} \cosh \left(r \sqrt{s / K_{g}}\right)}{r \sinh \left(a \sqrt{s / K_{g}}\right)}-\frac{a \sinh \left(r \sqrt{s / K_{g}}\right)}{r^{2} \sinh \left(a \sqrt{s / K_{g}}\right)}
$$

hence,

$$
\frac{\partial \phi}{\partial r}(a, s)=\sqrt{\frac{s}{K_{g}}} \operatorname{coth}\left(a \sqrt{s / K_{g}}\right)-\frac{1}{a} .
$$

Thus, (11) becomes

$$
\bar{F}(x, s)=\frac{3 \kappa_{g}\left(1-\phi_{a}\right)}{a} \bar{T}_{a}(x, s)\left[\sqrt{\frac{s}{K_{g}}} \operatorname{coth}\left(a \sqrt{s / K_{g}}\right)-\frac{1}{a}\right] .
$$

Therefore, for (9) we have

$$
\begin{aligned}
& \frac{\partial^{2} \bar{T}_{a}}{\partial x^{2}}= \\
& \bar{T}_{a}\left[\frac{1}{K_{a}}\left(s+\frac{3 \kappa_{g}\left(1-\phi_{a}\right)}{a \rho_{a} c_{a}}\left[\sqrt{\frac{s}{K_{g}}} \operatorname{coth}\left(a \sqrt{s / K_{g}}\right)-\frac{1}{a}\right]\right)\right] .
\end{aligned}
$$

Now, using the transformed boundary conditions

$$
\bar{T}_{a}(0, s)=\frac{T_{b}}{s}, \quad \lim _{x \rightarrow \infty} \bar{T}_{a}(x, s)=0,
$$

we can easily find the solution to (15) to be

$$
\bar{T}_{a}(x, s)=\frac{T_{b}}{s} e^{-x \sqrt{p}},
$$

and from (13) we have

$$
\bar{T}_{g}(x, r, s)=\frac{T_{b}}{s} e^{-x \sqrt{p}} \frac{a \sinh \left(r \sqrt{s / K_{g}}\right)}{r \sinh \left(a \sqrt{s / K_{g}}\right)},
$$


where

$$
p=\frac{1}{K_{a}}\left(s+\frac{3 \kappa_{g}\left(1-\phi_{a}\right)}{a \rho_{a} c_{a}}\left[\sqrt{\frac{s}{K_{g}}} \operatorname{coth}\left(a \sqrt{s / K_{g}}\right)-\frac{1}{a}\right]\right) .
$$

Inversions of equations (16) and (17) cannot be done analytically, so in the next section we obtain small and large time approximations to them.

\subsection{Small and large time analytical approximations}

To obtain semi-analytical solutions to the inverse Laplace transforms of (16) and (17), we consider small and large time approximations.

We begin by considering small time approximations, which corresponds to the limit as $s \rightarrow \infty$.

Now,

$$
\lim _{s \rightarrow \infty} p=\frac{1}{K_{a}}\left[s+\frac{3 \kappa_{g}\left(1-\phi_{a}\right)}{a \rho_{a} c_{a}}\left(\frac{\sqrt{s}}{\sqrt{K_{g}}}-\frac{1}{a}\right)\right]
$$

as $\lim _{s \rightarrow \infty} \operatorname{coth}(\mathrm{s})=1$.

Hence,

$$
\lim _{s \rightarrow \infty} p=\frac{1}{K_{a}}\left(s+m_{1} \sqrt{s}-m_{2}\right)
$$

where

$$
m_{1}=\frac{3 \kappa_{g}\left(1-\phi_{a}\right)}{a \rho_{a} c_{a} \sqrt{K_{g}}}
$$

and

$$
m_{2}=\frac{3 \kappa_{g}\left(1-\phi_{a}\right)}{a^{2} \rho_{a} c_{a}} .
$$

Now,

$$
\begin{aligned}
\left(s+m_{1} \sqrt{s}-m_{2}\right)^{1 / 2} & =s^{1 / 2}\left[1+\left(\frac{m_{1}}{\sqrt{s}}-\frac{m_{2}}{s}\right)\right]^{1 / 2} \\
& \approx s^{1 / 2}\left[1+\frac{1}{2}\left(\frac{m_{1}}{\sqrt{s}}-\frac{m_{2}}{s}\right)\right] \\
& =s^{1 / 2}+\frac{m_{1}}{2}-\frac{m_{2}}{2 \sqrt{s}} \\
& \approx s^{1 / 2}+\frac{m_{1}}{2} .
\end{aligned}
$$


Hence,

$$
\begin{aligned}
\lim _{s \rightarrow \infty} \bar{T}_{a}(x, s) & \approx \frac{T_{b}}{s} \exp \left\{-\frac{x}{\sqrt{K_{a}}}\left(s^{1 / 2}+\frac{m_{1}}{2}\right)\right\} \\
& =\frac{T_{b}}{s} \exp \left\{-\frac{x}{\sqrt{K_{a}}} s^{1 / 2}\right\} \exp \left\{-\frac{x m_{1}}{2 \sqrt{K_{a}}}\right\} .
\end{aligned}
$$

Now,

$$
\begin{aligned}
\lim _{s \rightarrow \infty} \frac{\sinh \left(\sqrt{s / K_{g}}\right) a}{\sinh \left(a \sqrt{s / K_{g}}\right) r} & =\frac{1 / 2\left(e^{r \sqrt{s / K_{g}}}-e^{-r \sqrt{s / K_{g}}}\right) a}{1 / 2\left(e^{a \sqrt{s / K_{g}}}-e^{-a \sqrt{s / K_{g}}}\right) r} \\
& \approx \frac{a}{r} e^{(r-a) \sqrt{s / K_{g}}}
\end{aligned}
$$

Hence,

$$
\begin{aligned}
\lim _{s \rightarrow \infty} \bar{T}_{g}(a, r, s) & =\frac{T_{b}}{s} \exp \left\{-\frac{x}{\sqrt{K_{a}}} s^{1 / 2}\right\} \exp \left\{\frac{x m_{1}}{2 \sqrt{K_{a}}}\right\} \frac{a}{r} e^{(r-a) \sqrt{s / K_{g}}} \\
& =\frac{a T_{b}}{r s} \exp \left\{-\frac{x m_{1}}{2 \sqrt{K_{a}}}\right\} \exp \left\{\left(\frac{(r-a)}{\sqrt{K_{g}}}-\frac{x}{\sqrt{K_{a}}}\right) \sqrt{s}\right\} .
\end{aligned}
$$

Upon inverting, we get

$$
\lim _{t \rightarrow 0} T_{a}(x, t)=T_{b} \exp \left\{-\frac{3 x \kappa_{g}\left(1-\phi_{a}\right)}{2 a \rho_{a} c_{a} \sqrt{K_{g}} \sqrt{K_{a}}}\right\} \operatorname{erfc}\left\{\frac{x}{2 \sqrt{K_{a}} \sqrt{t}}\right\},
$$

and

$$
\begin{aligned}
& \lim _{t \rightarrow 0} T_{g}(x, r, t)= \\
& \quad \frac{a T_{b}}{r} \exp \left\{\frac{-3 x \kappa_{g}\left(1-\phi_{a}\right)}{2 a \rho_{a} c_{a} \sqrt{K_{g}} \sqrt{K_{a}}}\right\} \operatorname{erfc}\left\{-\frac{1}{2 \sqrt{t}}\left(\frac{(r-a)}{\sqrt{K_{g}}}-\frac{x}{\sqrt{K_{a}}}\right)\right\} .
\end{aligned}
$$

We now consider large time approximations, which corresponds to the limit as $s \rightarrow 0$. Now, for small $x, \operatorname{coth}(\mathrm{x}) \approx 1 / x+x / 3+\mathrm{O}\left(x^{3}\right)$, hence

$$
\begin{aligned}
& \lim _{s \rightarrow 0} p \\
& =\frac{1}{K_{a}}\left(s+\frac{3 \kappa_{g}\left(1-\phi_{a}\right)}{a \rho_{a} c_{a}}\left[\sqrt{\frac{s}{K_{g}}}\left(\frac{1}{a \sqrt{s / K_{g}}}+\frac{a \sqrt{s / K_{g}}}{3}\right)-\frac{1}{a}\right]\right) \\
& =\frac{1}{K_{a}}\left(s\left[1+\frac{\kappa_{g}\left(1-\phi_{a}\right)}{K_{g} \rho_{a} c_{a}}\right]\right)
\end{aligned}
$$


thus,

$$
\lim _{s \rightarrow 0} \bar{T}_{a}(x, s)=\frac{T_{b}}{s} \exp \left\{-x s^{1 / 2}\left(\frac{1}{K_{a}}\left[1+\frac{\kappa_{g}\left(1-\phi_{a}\right)}{K_{g} \rho_{a} c_{a}}\right]\right)^{1 / 2}\right\} .
$$

Now,

$$
\begin{aligned}
\lim _{s \rightarrow 0} \frac{a \sinh \left(r \sqrt{s / K_{g}}\right)}{r \sinh \left(a \sqrt{s / K_{g}}\right)} & =\frac{a\left(e^{r \sqrt{s / K_{g}}}-e^{-r \sqrt{s / K_{g}}}\right)}{r\left(e^{a \sqrt{s / K_{g}}}-e^{-a \sqrt{s / K_{g}}}\right)} \\
& =\frac{0}{0},
\end{aligned}
$$

so that from L'Hopital's Rule we find

$$
\lim _{s \rightarrow 0} \frac{a\left(r / \sqrt{K_{g}} 1 / 2 s^{-1 / 2} \cosh \left(r \sqrt{s / K_{g}}\right)\right)}{r\left(a / \sqrt{K_{g}} 1 / 2 s^{-1 / 2} \cosh \left(a \sqrt{s / K_{g}}\right)\right)}=1,
$$

hence,

$$
\lim _{s \rightarrow 0} \bar{T}_{g}(a, r, s)=\lim _{s \rightarrow 0} \bar{T}_{a}(x, s) .
$$

Upon inverting, we get

$$
\begin{aligned}
\lim _{s \rightarrow 0} \bar{T}_{g}(a, r, s) & =\lim _{s \rightarrow 0} \bar{T}_{a}(x, s) \\
& =T_{b} \operatorname{erfc}\left\{\frac{x}{2 \sqrt{t}}\left(\frac{1}{K_{a}}\left[1+\frac{\kappa_{g}\left(1-\phi_{a}\right)}{\rho_{a} c_{a} K_{g}}\right]\right)^{1 / 2}\right\} .
\end{aligned}
$$

We observe that for large times, the air and grain temperatures are equivalent due to equilibrium (steady state temperature) being reached, that is, $T_{a}=T_{g}=T_{b}$ as limit $t \rightarrow \infty$.

\subsection{Some results}

As the performance of the Stehfest algorithm is well known for diffusion problems, we use it to determine the inverse Laplace transforms of $\bar{T}_{a}(x, s)$ and $\bar{T}_{g}(x, r, s)$, equations (16) and (17).

The following is a list of the parameter values used: $T_{b}=10^{\circ} \mathrm{C}, T_{a 0}=0^{\circ} \mathrm{C}$, $T_{g 0}=0^{\circ} \mathrm{C}, K_{a}=2.2 \mathrm{E}-05 \mathrm{~m}^{2} \mathrm{~s}^{-1}, K_{g}=8.3 \mathrm{E}-08 \mathrm{~m}^{2} \mathrm{~s}^{-1}, \rho_{a}=1.177 \mathrm{Kgm}^{-3}$, $c_{a}=1005 \mathrm{JK}_{\mathrm{g}}^{-1{ }^{\circ}} \mathrm{C}^{-1}, \kappa_{g}=0.001 \mathrm{Jm}^{-1} \mathrm{~s}^{-1{ }^{\circ}} \mathrm{C}^{-1}, a=0.015 \mathrm{~m}, r=0.0001 \mathrm{~m}$, $\phi_{a}=0.6, N_{p}=18$ (the Stehfest parameter). 
The scenario we envisage is that of a silo wall being heated by ambient temperature $T_{b}$ with the air and grain at some initial temperature, $T_{a 0}$ and $T_{g 0}$, respectively.

Results pertaining to large time approximations are not illustrated as they equal the boundary temperature $T_{b}$, as has been shown analytically in the previous section, and agreement is found to be exact, to graphical accuracy. Results illustrating the agreement between the numerical and semi-analytical solutions for small and large time are also not shown as the agreement is found to be exact, to graphical accuracy.

Figure 1 illustrates the variation of the numerical temperatures $T_{a}(-)$ and $T_{g}(--)$ with distance up to 30 seconds. There is a difference between the two, with the heat penetrating noticeably further through the air than the grain after the same amount of time has elapsed.

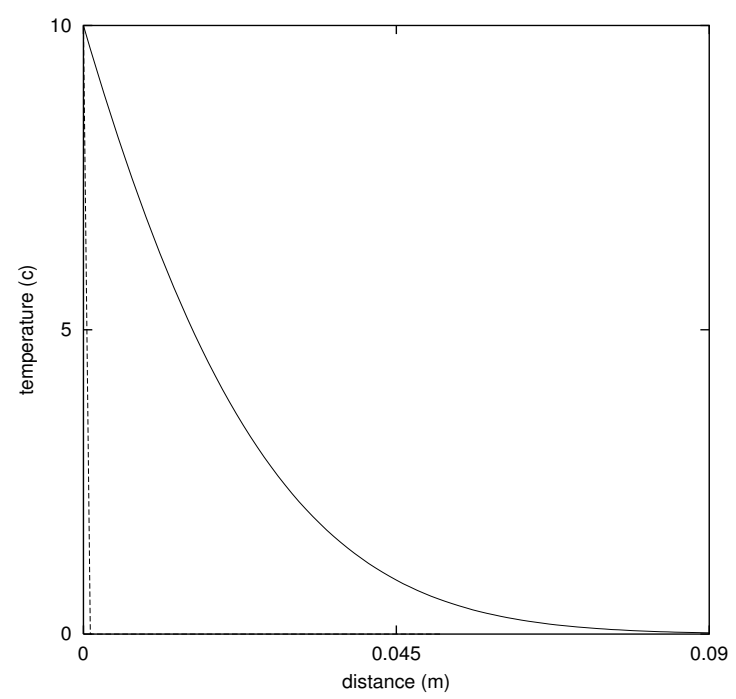

Figure 1. Numerical temperatures $T_{a}(-)$ and $T_{g}(--)$ vs distance up to 30 seconds

Figure 2 illustrates the variation of the temperature profiles of $T_{a}$ and $T_{g}$, up to 1000 seconds at a distance of $0.002 \mathrm{~m}$ from the wall. It illustrates the change in shape with time of the temperature profiles of air and grain at the point $x=0.002 \mathrm{~m}$. Due to the lag between the two, the temperature of the air approaches the steady-state temperature faster, as expected. Also, as a result of the two temperatures being modelled differently, their profiles are different in shape. Furthermore, the model predicts that as time 
increases, the air and grain temperatures reach a steady-state temperature, the boundary temperature $T_{b}$.

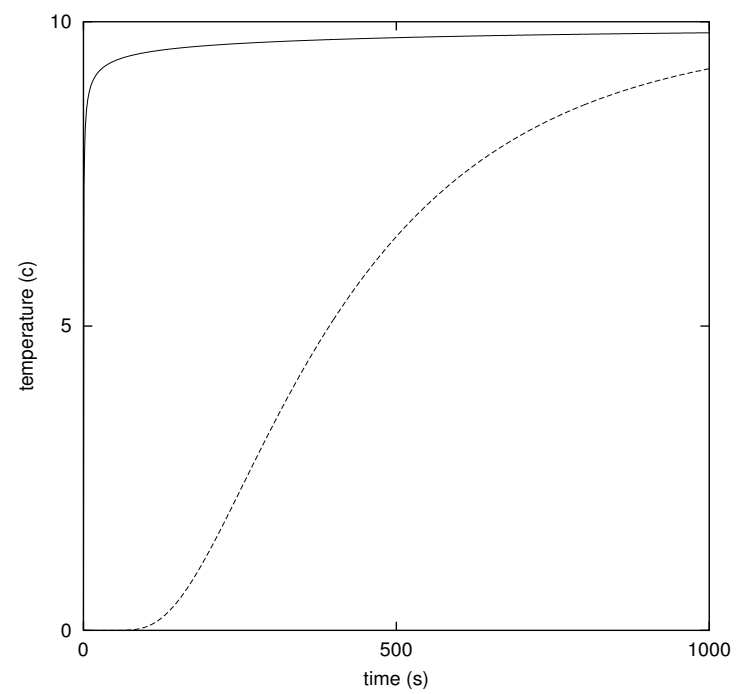

Figure 2. Numerical temperatures $T_{a}(-)$ and $T_{g}(--)$ vs time at $x=0.002 \mathrm{~m}$

\section{Two-Stage Model Versus Double-Diffusivity Model}

Antic and Hill [1] developed a double-diffusivity heat transfer model for predicting temperatures in grain stores, and in particular, within the peripheral layer. The double-diffusivity heat transfer model in its most general form is:

$$
\begin{aligned}
& \frac{\partial T_{a}}{\partial t}=K_{a} \frac{\partial^{2} T_{a}}{\partial x^{2}}-k_{1} T_{a}+k_{2} T_{g}, \\
& \frac{\partial T_{g}}{\partial t}=K_{g} \frac{\partial^{2} T_{g}}{\partial x^{2}}+k_{3} T_{a}-k_{4} T_{g},
\end{aligned}
$$

where $k_{1}=k_{a} /\left(\rho_{a} c_{a}\right), k_{2}=k_{g} /\left(\rho_{a} c_{a}\right), k_{3}=k_{a} /\left(\rho_{g} c_{g}\right)$, and $k_{4}=k_{g} /\left(\rho_{g} c_{g}\right)$. A semi-analytical solution was obtained by the Heat Balance Integral Method (HBIM), and was found to be in very good agreement with an explicit FTCS finite difference numerical solution. We note that it can be shown that $X_{i} \approx c_{i} \sqrt{t}$, for $i=a, g$ and $c$ constant, where $X_{i}$ represents the penetration depth through the air or grain path. 
The model is based on a heat transfer variant of the theory of doublediffusivity as proposed by Hill [8]. The double-diffusivity model originates from Barenblatt et al [3]. Both the two-stage model and the doublediffusivity model are dual region models in that they identify air and grain temperatures separately within the grain bulk. The microscopic configuration of the grain bulk is important to both models for obtaining a welldefined macroscopic description, but they differ in how they identify this microstructure. The fundamental assumption behind the double-diffusivity model is that there exist two connection pathways such that both the air and grain occupy each point within the grain bulk, with the temperature of that point being the average of the two temperatures. Heat transfer takes place along either the air path or the grain path, with the possibility of the heat being transferred from one path to the other. The main problem with such an assumption, as outlined in Davis and Hill [5] who reviewed the approaches of Barenblatt [3] and McNabb [10] for the modelling of flow in fissured rocks by considering their suitability to particular problems, is that it assumes that any infinitesimally small volume of the grain bulk is occupied by both air and grain, meaning that it is impossible to know whether a particular point lies within either the air or the grain. The two-stage model does not suffer such a problem. It too considers a double-porosity mechanism, but uses a greater level of detail for the temperature of the grain particles. In the double-diffusivity model the grain particle is treated as a lump with one uniform temperature throughout, whereas the two-stage model identifies a separate spatial coordinate system with the grain, that is, it considers the variation in temperature with distance from the surface of the grain kernel.

We now compare the numerical results obtained for the double-diffusivity heat transfer model [1], with the numerical results of the two-stage heat transfer model. The volume-averaged grain temperature is used. The same parameter values are used as in Section 4.2, along with $\rho_{g}=513, c_{g}=1380$, and $k_{a}=0.01, k_{g}=0.01$.

Figure 3 illustrates the variation of the two-stage and double-diffusivity numerical temperatures $T_{a},(-)$ and $(--)$, and $T_{g},(--)$ and $(\cdots)$, respectively, vs distance up to 30 seconds. We observe that both the air and grain temperatures of the two-stage model lag behind the double-diffusivity heat transfer model. This is due to the fundamental differences in how the two models define the air and grain temperatures. Both models predicts that there exists a difference in the air and grain temperatures, with one lagging the other. In Figure 4 we observe that this lag decreases as time increases, and hence, the two models effectively predict the same values. 


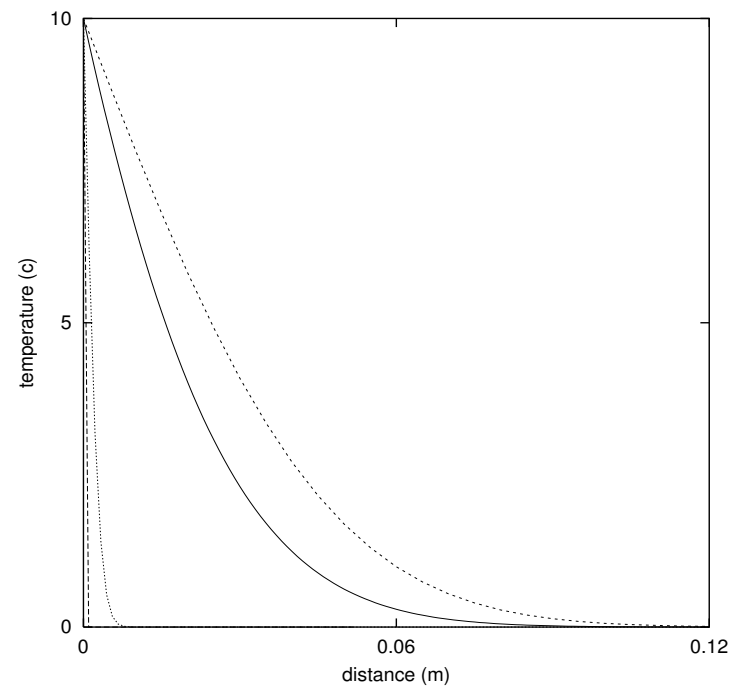

Figure 3. Two-stage and double-diffusivity numerical temperatures $T_{a},(-)$ and (- -), and $T_{g},(--)$ and $(\cdots)$, respectively, vs distance up to 30 seconds

Figure 4 illustrates the variation of the two-stage and double-diffusivity numerical temperatures $T_{a},(-)$ and $(--)$, and $T_{g},(--)$ and $(\cdots)$, respectively, up to 700 seconds at a distance of $0.002 \mathrm{~m}$ from the wall. Once again, we observe that both the air and grain temperatures of the twostage model lag behind the double-diffusivity heat transfer model, which approach the steady-state temperature faster. We also note that the profile of the two-stage heat transfer model grain temperature prediction is different to that of the double-diffusivity heat transfer model. This is primarily due to the fundamental differences in how the two models define the grain temperature. Both models predict that as time increases, the air and grain temperatures reach a steady-state temperature, the boundary temperature $T_{b}$.

\section{Conclusions}

An understanding of the flow of heat in the peripheral layer between the grain bulk and the grain store wall is very important from many industrial perspectives. Here we have proposed using the two-stage heat transfer model, which takes into account the fact that the rate of heat transfer 


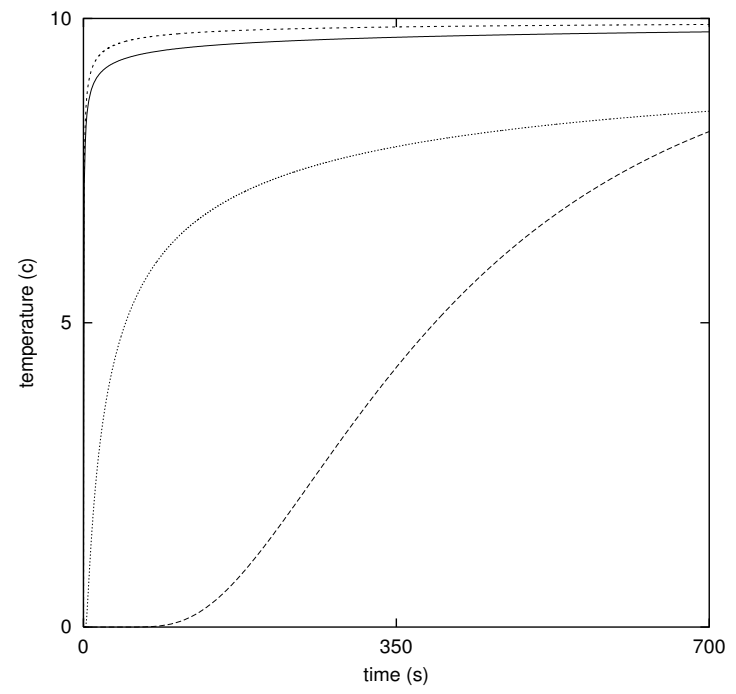

Figure 4. Two-stage and double-diffusivity numerical temperatures $T_{a},(-)$ and (- -), and $T_{g},(--)$ and $(\cdots)$, respectively, vs time at $x=0.002 \mathrm{~m}$

through the grain is different to that through the interstitial air surrounding it, and where the grains are assumed to be well-defined as spheres. On comparing the two-stage model with the previously proposed doublediffusivity model of the authors [1], we find that models predict that there exists a difference in the air and grain temperatures of the grain bulk. We observe that the predictions of the air and grain temperatures of the twostage model lag behind those of the double-diffusivity heat transfer model, but this lag decreases as time increases. This is related to the fundamental differences existing between the two models, particularly, with how they define not only the microstructure of the air and grain, but the interaction between the air and grain. Investigating the temperature profiles within the peripheral layer via experimentation is difficult due to the small scales involved, and it is even more difficult to measure the air and grain temperatures separately. The main motivation behind this modelling is the lack of experimental data which is currently available. The results obtained are physically reasonable, and at least provide some insight into the potential differences existing between the air and grain temperatures. 


\section{Acknowledgments}

This work is undertaken as part of a collaborative research project with the Stored Grain Research Laboratory, CSIRO Division of Entomology, ACT, Australia. The authors are especially grateful to Dr Alex McNabb, Dr Mark McGuiness, Dr Tim Marchant and Mr James Darby for many helpful discussions, The first author (AA) gratefully acknowledges the Bulk Handling Companies of Australia for a Ph.D. Scholarship, and the second author (JMH) wishes to acknowledge the Australian Research Council for provision of a Senior Research Fellowship.

\section{References}

1. A. Antic and J. M. Hill. A Mathematical Model for Heat Transfer in Grain Store Microclimates. ANZIAM J. (E), 42:C117-C133, 2000.

2. R. E. Babcock, D. W. Green, and R. H. Perry. Longitudinal Dispersion Mechanisms in Packed Beds. A.I.Ch.E. Journal, 12:922-927, 1966.

3. G. I. Barenblatt, Iu. P. Zheltov, and I. N. Kochina. Basic Concepts in the Theory of Seepage of Homogeneous Liquids in Fissured Rocks. J. Appl. Math. Mech., 24:1286-1303, 1960.

4. B. Davies and B. Martin. Numerical Inversion of the Laplace Transform: A Survey and Comparison of Methods. J. Comp. Phys., 33:1-32, 1979.

5. G. B. Davis and J. M. Hill. A Review of Flow in Fissured Rocks and Related Phenomena. Mech. Res. Comm., 9:115-120, 1982.

6. G. B. Davis. Mathematical Modelling of Rate-Limiting Mechanisms of Pyritic Oxidation in Overburden Dumps. PhD Thesis, University of Wollongong, 1983.

7. R. Gudhe, K. R. Rajagopal, M. Massoudi, and R. C. Yalamanchili. Gravity Flow of a Fluid-Particle Mixture in a Channel. Developments in Fluidization and FluidParticle Systems, AIChE Symposium Series, 308:112-118, 1995.

8. J. M. Hill. A Discrete Random Walk Model for Diffusion in Media with Double Diffusivity. J. Austral. Math. Soc. (Series B), 22:58-74, 1980.

9. M. J. McGuinness. Pressure Transmission in a Bounded Randomly Fractured Reservoir of Single-Phase Fluid. Trans. in Por. Med., 1:371-397, 1986.

10. A. McNabb. Factorizable 'Fruit Cake' Boundary Value Problems. Suppl. Newsletter No. 12 N.Z. Math. Soc., 9-18. 1978.

11. A. E. Milne. The Diffusion of Imprisoned Radiation Through a Gas. J. London Math. Soc., 40-51, 1926.

12. A. F. Moench. Double-Porosity Models for a Fissured Groundwater Reservoir with Fracture Skin. Water Resources. Res., 20:831-846, 1984.

13. K. Pruess. A Practical Method for Modelling Fluid and Heat Flow in Fractures Porous Media. Soc. Pet. Eng. J., 14-26, 1985.

14. J. Skopp and A. W. Warrick. A Two-Phase Model for the Miscible Displacement of Reactive Solutions in Soils. Proc. Soil Sc. Soc. Am., 38:545-550, 1974.

15. H. Stehfest. Algorithm 368: Numerical Inversion of Laplace Transform. Comm. ACM, 13:47-49, 1970 .

16. V. W. Uhl and W. L. Root. Heat Transfer to Granular Solids in Agitated Units. Chem. Eng. Prog., 63:81-92, 1967. 
17. W. L. Vargas and J. J. McCarthy. Unsteady Heat Conduction in Granular Materials. Mat. Res. Soc. Symp. Proc., Materials Research Society, 627:BB3.9.1-BB3.9.6, 2000.

18. W. L. Vargas and J. J. McCarthy. Heat Conduction in Granular Materials. AIChE. J., 47:1052-1059, 2001.

19. D. Vortmeyer and R. J. Schaefer. Equivalence of One- and Two-Phase Models for Heat Transfer Processes in Packed Beds: One Dimensional Theory. Chem. Eng. Sci., 29:485-491, 1973.

20. J. E. Warren and P. J. Root. The Behaviour of Naturally Fractured Reservoirs. Soc. Pet. Eng. J., 245-255, 1963.

21. G. J. Weir. Single-Phase Flow Regimes in a Discrete Fracture Model. Water Res. Res., 65-73, 1999.

22. S. Zhu, P. Satravaha, and X. Lu. Solving Linear Diffusion Equations with the Dual Reciprocity Method in Laplace Space. Engng. Anal. with Boundary Element, 13:1-10, 1994. 


\title{
Nomenclature
}

\author{
$T_{a}$ is the temperature of air \\ $T_{g}$ is the temperature of grain \\ $K_{a}$ is the thermal diffusivity of air \\ $K_{g}$ is the thermal diffusivity of grain \\ $k_{a}$ is the effective heat transfer coefficient of air \\ $k_{g}$ is the effective heat transfer coefficient of grain \\ $\kappa_{a}$ is the thermal conductivity of air \\ $\kappa_{g}$ is the thermal conductivity of grain \\ $\rho_{a}$ is the density of air \\ $\rho_{g}$ is the density of grain \\ $c_{a}$ is the specific heat of air at constant pressure \\ $c_{g}$ is the specific heat of grain at constant pressure \\ $T_{a 0} \quad$ is the initial temperature of the air \\ $T_{g 0}$ is the initial temperature of the grain \\ $T_{b}(t)$ is the boundary temperature \\ $r$ is the spatial coordinate system in the grain \\ $a$ is the radius of the grain \\ $\Phi_{a}$ is the fraction of grain bulk volume occupied by air \\ $P_{f}(x, t)$ is the fluid pressure in the fissure system at position \\ $x$ at time $t$ \\ $P_{b}(x, y, t)$ is the pressure change in the block at position $y$ at \\ time $t$ located in the neighbourhood of position $x$ \\ $\Phi_{f} \quad$ is the porosity of the fissure system \\ $\Phi_{b} \quad$ is the porosity of the blocks \\ $\mu$ is the dynamic viscosity of the fluid \\ $c$ is the compressibility of the fluid at constant pressure \\ $K_{f} \quad$ is the permeability of the fissure system \\ $K_{b} \quad$ is the permeability of the blocks \\ $V_{x}$ is the volume of block in the neighbourhood of $x$ \\ $S_{x}$ is the surface of the block in the neighbourhood of $x$
}




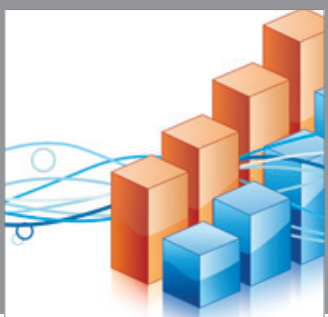

Advances in

Operations Research

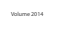

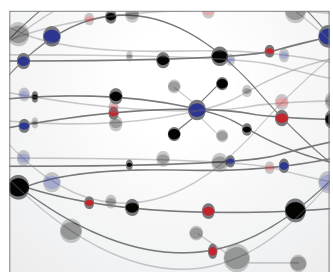

\section{The Scientific} World Journal
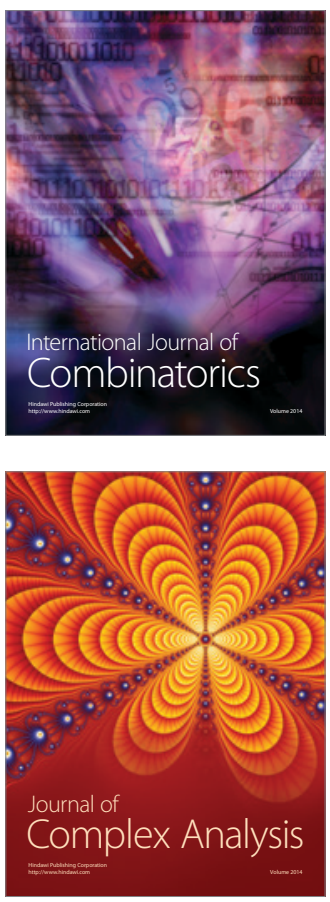

International Journal of

Mathematics and

Mathematical

Sciences
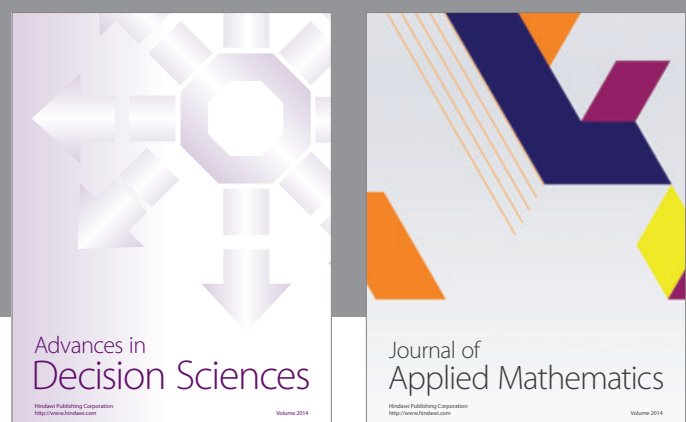

Journal of

Applied Mathematics
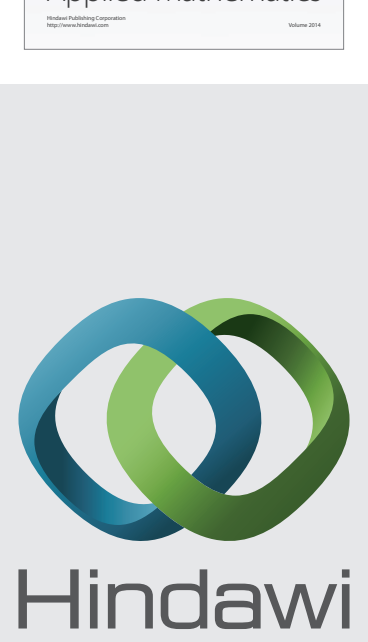

Submit your manuscripts at http://www.hindawi.com
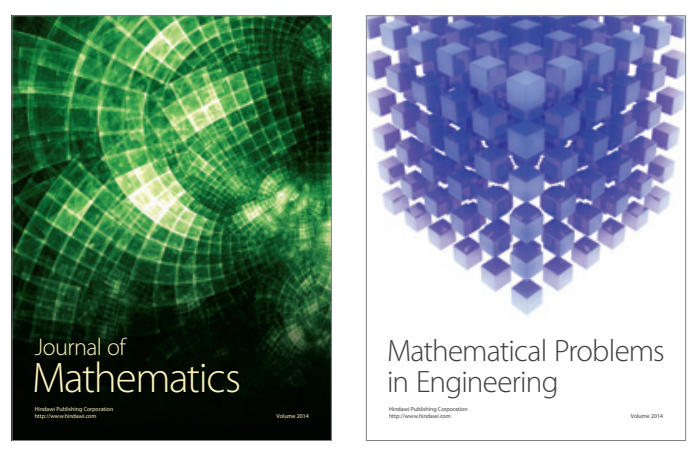

Mathematical Problems in Engineering
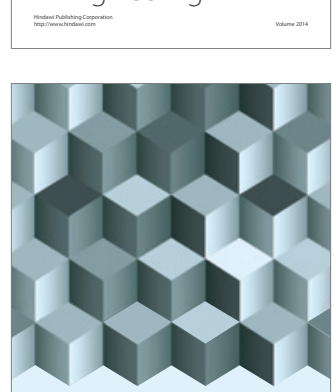

Journal of

Function Spaces
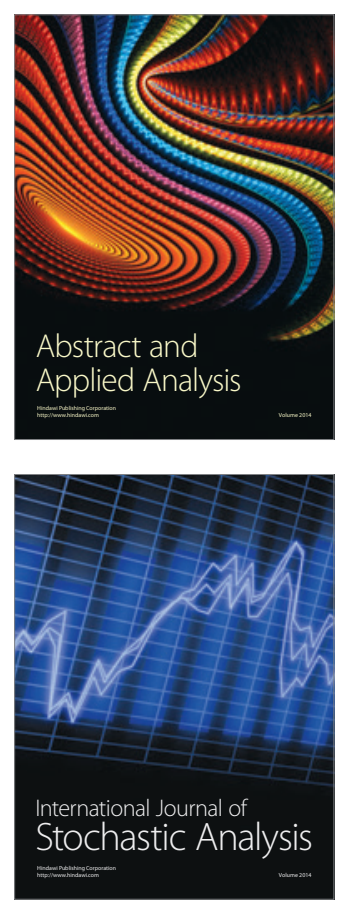

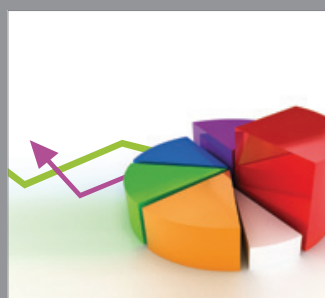

ournal of

Probability and Statistics

Promensencen
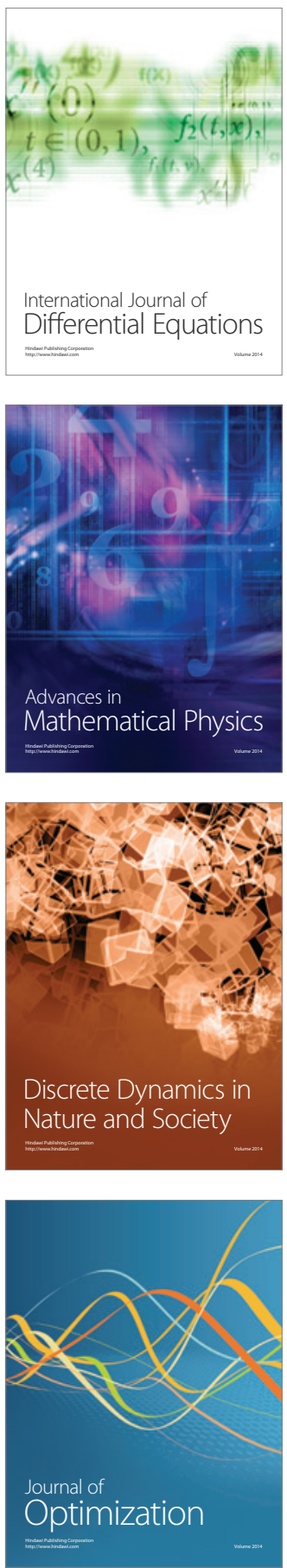